\title{
Assédio sexual sofrido por profissionais de enfermagem nas instituições de saúde
}

\author{
Sexual harassment suffered by nursing professionals in healthcare instituitions \\ El acoso sexual sufrido por los profesionales de enfermeira em las instituciones de salud
}

Recebido: 26/06/2021 | Revisado: 04/07/2021 | Aceito: 19/07/2021 | Publicado: 27/07/2021

\author{
Rayssa do Nascimento Sousa \\ ORCID: https://orcid.org/0000-0001-7865-1637 \\ Universidade Estadual do Piauí, Brasil \\ E-mail: rayssasousa@aluno.uespi.br \\ Vitoria Vilas Boas da Silva Bomfim \\ ORCID: https://orcid.org/0000-0003-4897-0279 \\ Centro Universitário Jorge Amado, Brasil \\ E-mail: pesquisaclinica9@gmail.com \\ Ághata Monike Paula da Silva Lins \\ ORCID: https://orcid.org/0000-0002-4606-8209 \\ Centro Universitário Brasileiro, Brasil \\ E-mail: aghatamonike@ hotmail.com \\ Catarina Vilas Boas da Silva Bomfim \\ ORCID: https://orcid.org/0000-0002-5537-8356 \\ Universidade Salvador, Brasil \\ E-mail: vilasboascatarina@gmail.com \\ Andressa Fernandes da Silva \\ ORCID: https://orcid.org/0000-0002-1942-0860 \\ Instituto Nacional de Cardiologia, Brasil \\ E-mail: andressafdurso@gmail.com \\ Maria Eduarda Wanderley de Barros Silva \\ ORCID: https://orcid.org/0000-0002-4642-3282 \\ Universidade Federal de Campina Grande, Brasil \\ E-mail: eduarda.wanderley@ outlook.com
}

\begin{abstract}
Resumo
Objetivo: identificar nas publicações científicas as características e desfechos do assédio sexual sofrido por profissionais de enfermagem nas instituições de saúde. Método: Revisão integrativa, realizada no mês de junho de 2021. A princípio, foram identificados nas bases de dados 112 publicações. Após aplicar os critérios de inclusão foram encontrando 23 artigos, que foram lidos na íntegra. Aplicados os critérios de exclusão, obtendo após análise criteriosa uma amostra de nove artigos. Resultados: As vítimas mais frequentes, foram as mulheres jovens, solteiras, e com pouco tempo de atuação. Entre os apontados como perpetradores de assédio sexual, a maioria era homem, paciente e/ou familiar, médico, outro enfermeiro, e os profissionais que exerciam cargos de chefia. Entre os obstáculos para não denunciar estão, os sentimentos de vergonha e constrangimento, desacreditar que haveria sanções aos assediadores, e as dificuldades em realizar as denúncias. Os enfermeiros apresentaram elevados níveis de estresse, expressaram tristeza, e tiveram lembranças perturbadoras sobre o momento do assédio. Conclusões: Diante do exposto faz necessário o encorajamento dos profissionais e o treinamento destes para conseguirem identificar essas situações, e agir corretamente denunciando. Além disso, se faz necessário não normalizar as situações difíceis que muitas vezes os profissionais deixam passar em função do trabalho.
\end{abstract}

Palavras-chave: Enfermagem; Assédio sexual; Violência no trabalho.

\begin{abstract}
Objective: to identify in scientific publications the characteristics and outcomes of sexual harassment suffered by nursing professionals in health institutions. Method: Integrative review, carried out in June 2021. At first, 112 publications were identified in the databases. After applying the inclusion criteria, 23 articles were found, which were read in full. Exclusion criteria were applied, obtaining after careful analysis a sample of nine articles. Results: The most frequent victims were young women, single, and with little time of experience. Among those identified as perpetrators of sexual harassment, most were men, patients and/or family members, physicians, other nurses, and professionals who held leadership positions. Among the obstacles to not reporting are feelings of shame and embarrassment, disbelieving that there would be sanctions against harassers, and difficulties in reporting. Nurses showed high levels of stress, expressed sadness, and had disturbing memories about the moment of harassment. Conclusions: Given the above, it is necessary to encourage professionals and train them to be able to identify these situations, and act correctly reporting. In addition, it is necessary not to normalize the difficult situations that professionals often let go of because of their work.
\end{abstract}


Keywords: Nursing; Sexual harassment; Violence at work.

\section{Resumen}

Objetivo: identificar en publicaciones científicas las características y desenlaces del acoso sexual que sufren los profesionales de enfermería en las instituciones de salud. Método: Revisión integrativa, realizada en junio de 2021. En un primer momento se identificaron 112 publicaciones en las bases de datos. Después de aplicar los criterios de inclusión, se encontraron 23 artículos, los cuales fueron leídos en su totalidad. Se aplicaron criterios de exclusión, obteniendo tras un cuidadoso análisis una muestra de nueve artículos. Resultados: Las víctimas más frecuentes fueron mujeres jóvenes, solteras y con poco tiempo de experiencia. Entre los identificados como autores de acoso sexual, la mayoría eran hombres, pacientes y / o familiares, médicos, otras enfermeras y profesionales que ocupaban puestos de liderazgo. Entre los obstáculos para no denunciar están los sentimientos de vergüenza y vergüenza, la incredulidad de que habría sanciones contra los acosadores y las dificultades para denunciar. Las enfermeras mostraban altos niveles de estrés, expresaban tristeza y tenían recuerdos perturbadores sobre el momento del acoso. Conclusiones: Dado lo anterior, es necesario incentivar y capacitar a los profesionales para que sean capaces de identificar estas situaciones y actuar correctamente informando. Además, es necesario no normalizar las situaciones difíciles que los profesionales suelen dejar ir a causa de su trabajo.

Palabras clave: Enfermería; El acoso sexual; Violencia en el trabajo.

\section{Introdução}

Sofrer violência durante o trabalho é realidade para muitos profissionais, que passam a desenvolver suas atividades em meio a condições laborais desfavoráveis. Entre os tipos de violência ocupacional, estão, a agressão verbal, assédio moral, violência física, assédio sexual e discriminação racial. Além da violência que adentra os serviços de saúde, como expressão da criminalidade no território (Pedro et al., 2017; Busnello et al., 2021; Bordignon \& Monteiro, 2021; Lisboa et al., 2016).

A magnitude do impacto da violência na vida e carreira desses profissionais ainda é pouco discutida, podendo assim passar despercebida, no entanto, pode causar danos irreparáveis a autoestima das vítimas, e a convivência em um ambiente repressor, pode segundo Pedro et al. (2017) amedrontar e gerar problemas de saúde físicos e mentais nos profissionais de saúde, podendo levar a insatisfação com o trabalho (Correia, 2016; Marques \& Silva, 2017).

Diante desse contexto, um estudo realizado com enfermeiras coreanas que trabalhavam em hospital psiquiátrico e estavam repetidamente sofrendo agressões demonstrou a relação entre sofrer violência e desenvolver síndrome de burnout (Seo et al., 2019). Essas alterações psíquicas, podem ter repercussões graves na qualidade de vida dos profissionais, segundo Batista et al. (2019) a síndrome de burnout pode afetar o indivíduo dentro do trabalho e na convivência social. Assim, se apresenta em forma de estresse, causado por situações complexas decorrentes ao trabalho (Fernandes et al., 2017; Silva et al., 2018; Machado et al., 2016).

Perante o exposto, este trabalho teve como objetivo identificar nas publicações científicas as características e desfechos do assédio sexual sofrido por profissionais de enfermagem nas instituições de saúde.

\section{Metodologia}

Refere-se a uma revisão integrativa descritiva da literatura, que segundo Botelho et al. (2011), possibilita ao pesquisador sistematizar a produção científica já existente em busca de evidências sobre determinado tema.

Realizada no mês de junho de 2021, norteada pela questão "Quais as características e desfechos do assédio sexual sofrido por profissionais de enfermagem nas instituições de saúde", que foi definida através da estratégia PICO: Problema/ Assédio sexual; Interesse/ profissionais de enfermagem; Contexto/ ambiente de trabalho (Santos et al., 2007).

A busca foi realizada nas de dados Base de dados de Enfermagem (BDENF), Índice Bibliográfico Español en Ciencias de la Salud (IBECS), Literatura Latino-Americana e do Caribe em Ciências da Saúde (LILACS), Medical Literature Analysis and Retrieval System Online (MEDLINE/ PubMed) e, na Scientific Electronic Library Online (SciELO). A Partir do cruzamento dos DeCS: "Enfermagem", “Assédio Sexual", "Violência no trabalho", associados ao operador booleano "AND". 


\section{Resultados e Discussão}

A princípio, foram identificados nas bases de dados 112 publicações. Após aplicar os critérios de inclusão: textos publicados nos últimos cinco anos, disponíveis na íntegra, e em todos os idiomas, encontrando 23 artigos, que foram lidos na íntegra. E, aplicados os critérios de exclusão: pesquisas secundárias, teses, e artigos que não respondiam à questão de pesquisa. Obtendo, após análise criteriosa uma amostra de nove artigos, como retratado no (Quadro 1).

Quadro 1 - Artigos selecionados para compor a amostra final da revisão, Brasil, 2021.

\begin{tabular}{|c|c|c|c|}
\hline $\begin{array}{l}\text { AUTOR, } \\
\text { ANO }\end{array}$ & PAÍS & TÍTULO & ACHADOS \\
\hline $\begin{array}{l}\text { Bernardes } \text { et al., } \\
2021\end{array}$ & Brasil & $\begin{array}{l}\text { Workplace violence among nursing } \\
\text { professionals. }\end{array}$ & $\begin{array}{l}\text { Na maioria dos casos, o assédio sexual é ignorado ou não } \\
\text { denunciado devido ao constrangimento. }\end{array}$ \\
\hline $\begin{array}{l}\text { Boafo et al., } \\
2016\end{array}$ & Gana & $\begin{array}{l}\text { Sources, incidence and effects of non- } \\
\text { physical workplace violence against nurses } \\
\text { in Ghana. }\end{array}$ & $\begin{array}{l}\text { O assédio foi praticado por médicos, pacientes e seus } \\
\text { familiares; o assédio sexual ocorreu mais com mulheres, } \\
\text { jovens e solteiras. }\end{array}$ \\
\hline $\begin{array}{l}\text { Bordignon \& } \\
\text { Monteiro, } 2021\end{array}$ & Brasil & $\begin{array}{l}\text { Analysis of workplace violence against } \\
\text { nursing professionals and possibilities for } \\
\text { prevention. }\end{array}$ & $\begin{array}{l}\text { Os profissionais sofreram assédio por quatro vezes ou mais } \\
\text { em um ano; os eventos ocorreram principalmente à noite } \\
\text { pela manhã; pacientes, colegas de trabalho e chefes foram } \\
\text { os agressores principais; os perpetradores de assédio sexual } \\
\text { eram do sexo masculino e a vítima do sexo feminino. }\end{array}$ \\
\hline $\begin{array}{l}\text { Chang \& Cho, } \\
2016\end{array}$ & $\begin{array}{l}\text { Coreia do } \\
\quad \text { Sul }\end{array}$ & $\begin{array}{l}\text { Workplace Violence and Job Outcomes of } \\
\text { Newly Licensed Nurses }\end{array}$ & $\begin{array}{l}\text { Os pacientes, seguidos pelos familiares dos pacientes, } \\
\text { foram os principais perpetradores de assédio sexual, } \\
\text { seguidos por colegas enfermeiros e seu gerente de } \\
\text { enfermagem, respectivamente; Das } 15 \text { enfermeiras que } \\
\text { relataram assédio sexual por colegas enfermeiras, } 10 \text { eram } \\
\text { mulheres e } 5 \text { eram homens. }\end{array}$ \\
\hline $\begin{array}{l}\text { Honarvar et al., } \\
2019\end{array}$ & Irâ & $\begin{array}{l}\text { Violence against Nurses: A Neglected and } \\
\text { Health-threatening Epidemic in the } \\
\text { University Affiliated Public Hospitals in } \\
\text { Shiraz, Iran. }\end{array}$ & $\begin{array}{l}\text { A maioria dos enfermeiros não foram capacitados para o } \\
\text { enfrentamento da violência e seu controle no ambiente de } \\
\text { trabalho. } \\
\text { As piadas e brincadeiras de cunho sexual são o tipo de } \\
\text { violência mais comum relatado. }\end{array}$ \\
\hline Pai et al., 2018 & Brasil & $\begin{array}{l}\text { Violência física e psicológica perpetrada no } \\
\text { trabalho em saúde }\end{array}$ & $\begin{array}{l}\text { o assédio sexual havia se repetido para suas vítimas por } \\
\text { mais de três vezes no último ano. }\end{array}$ \\
\hline $\begin{array}{l}\text { Song et al., } \\
2021\end{array}$ & China & $\begin{array}{l}\text { Frequency and barriers of reporting } \\
\text { workplace violence in nurses: An online } \\
\text { survey in China. }\end{array}$ & $\begin{array}{l}\text { Quase metade dos enfermeiros afirmou que o hospital não } \\
\text { tinha sistema de notificação ou tinham dúvidas sobre o } \\
\text { sistema, e desconheciam como e quais tipos de violência } \\
\text { denunciar, as enfermeiras acreditam que o hospital dá mais } \\
\text { atenção aos pacientes do que à equipe }\end{array}$ \\
\hline $\begin{array}{l}\text { Tsukamoto et } \\
\text { al., } 2019\end{array}$ & Brasil & $\begin{array}{l}\text { Violência ocupacional na equipe de } \\
\text { enfermagem: prevalência e fatores } \\
\text { associados. }\end{array}$ & $\begin{array}{l}\text { O assédio sexual ocorreu duas vezes ou mais em } 1 \text { ano; Os } \\
\text { autores do assédio foram os colegas de trabalho, seguidos } \\
\text { dos chefes e supervisores e de pacientes e seus familiares; } \\
\text { do sexo masculino; associou-se a testemunhar esse tipo de } \\
\text { assédio e ser vítima de abuso verbal; foi maior no turno } \\
\text { noturno; e entre os mais jovens. }\end{array}$ \\
\hline
\end{tabular}

Fonte: Autores (2021).

Durante o trabalho os profissionais de enfermagem estiveram expostos ao assédio sexual, no entanto, as vítimas mais frequentes, foram as mulheres jovens, solteiras, e com pouco tempo de atuação. Entre os apontados como perpetradores de assédio sexual, a maioria era homem, paciente e/ou familiar, médico, enfermeiro, e profissionais que exerciam cargos de chefia (Boafo et al., 2016; Bordignon \& Monteiro, 2021; Chang \& Cho, 2016). Os assédios ocorreram principalmente no período da 
noite. Em forma de cantadas, piadas, histórias e contatos sexuais indesejados, que deixaram as vítimas constrangidas e sem conseguir sair da situação. Esses assédios se repetiram por mais de 2 vezes no ano anterior à participação nas pesquisas (Bordignon \& Monteiro, 2021; Bordignon \& Monteiro, 2016; Pedro et al., 2017; Pai et al., 2018; Honarvar et al., 2019; Tsukamoto et al., 2019).

Diante do assédio sexual, muitos profissionais não denunciam, para Tsukamoto et al. (2019) e Bernardes et al. (2021) entre os obstáculos para não denunciar estão, os sentimentos de vergonha e constrangimento, além, de desacreditar que haveria sanções aos assediadores. Esse ponto vem de encontro com as dificuldades em realizar as denúncias, que segundo Song et al. (2021) estão ligadas a não saber a quem denunciar, e no desconhecimento da existência dos sistemas de notificação ou ainda por não saberem como funcionam os sistemas de notificação. Há a necessidade de disseminar informações sobre os veículos para denúncia, além de treinamento dos profissionais para utilizar essas ferramentas.

Segundo Boafo et al. (2016) as reações mais comuns das profissionais durante o assédio sexual era dialogar com o perpetrador para que não continuasse com a prática do assédio, este fato, pode ser indicativo, tentativa de contornar a situação, já que pode haver déficit de treinamento sobre como lidar com a violência, ou na maioria das vezes não haver auxílio ao profissional. Assim, se faz necessário, abordar os temas relacionados ao assédio no local de trabalho, de maneira sensível e aberta, possibilitando aos profissionais, esclarecer suas dúvidas e serem orientados sobre o que fazer quando a violência acontece, buscando evitar que haja, por parte dos profissionais a negação ou desconhecimento de estar sendo vítima de um crime.

Portanto, é importante o estabelecimento de redes de apoio à saúde mental dos trabalhadores nas instituições de saúde, pois as repercussões do assédio sexual na saúde dos enfermeiros podam segundo Boafo et al. (2016) levar a altos níveis de estresse e a sentimentos de tristeza, além de lembranças perturbadoras sobre o momento do assédio. Este fato aponta, o potencial irreversível que a violência ocasiona a saúde das vítimas, e o quão importante são as relações saudáveis de trabalho, na qualidade de vida dos indivíduos.

\section{Considerações Finais}

Através da pesquisa foi possível caracterizar os profissionais de enfermagem vítimas de assédio sexual no ambiente do trabalho, além dos assediadores. Destacou-se, a necessidade de falar sobre o assédio sexual no ambiente de trabalho, e sobre os veículos de denúncia. Diante do exposto faz necessário o encorajamento dos profissionais e o treinamento destes para que consigam identificar essas situações, e agir corretamente denunciando. Além da necessidade de criação e fortalecimento da rede de prevenção de violência e apoio a vítima de assédio sexual no ambiente de trabalho, para inibir a ação dos perpetradores. Deste modo, para que a enfermagem exerça suas funções com qualidade se faz necessário um ambiente de trabalho saudável, cooperativo e respeitoso. Além disso, se faz necessário não normalizar as situações difíceis que muitas vezes os profissionais deixam passar em função do trabalho.

\section{Referências}

Batista, K. O. B., dos Santos Santos, J. F., Santos, S. D. S., de Andrade Aoyama, E., \& Lima, R. N. (2019). Síndrome de burnout em enfermeiros: consequências na atividade profissional. Revista Brasileira Interdisciplinar de Saúde.

Bernardes, M. L. G., Karino, M. E., Martins, J. T., Okubo, C. V. C., Galdino, M. J. Q., \& Moreira, A. A. O. (2020). Workplace violence among nursing professionals. Revista brasileira de medicina do trabalho, 18(3), 250.

Boafo, I. M., Hancock, P., \& Gringart, E. (2016). Sources, incidence and effects of non-physical workplace violence against nurses in Ghana. Nursing open, 3(2), 99-109.

Bordignon, M., \& Monteiro, M. I. (2016). Violência no trabalho da Enfermagem: um olhar às consequências. Revista brasileira de Enfermagem, 69(5), 996999. 
Research, Society and Development, v. 10, n. 9, e32510917582, 2021

(CC BY 4.0) | ISSN 2525-3409 | DOI: htttp://dx.doi.org/10.33448/rsd-v10i9.17582

Bordignon, M., \& Monteiro, M. I. (2021). Analysis of workplace violence against nursing professionals and possibilities for prevention. Revista gaúcha de enfermagem, 42 .

Botelho, L. L. R., de Almeida Cunha, C. C., \& Macedo, M. (2011). O método da revisão integrativa nos estudos organizacionais. Gestão e sociedade, 5(11), 121-136.

Busnello, G. F., Trindade, L. D. L., Pai, D. D., Beck, C. L. C., \& Ribeiro, O. M. P. L. (2021). Tipos de violencia en el trabajo de enfermería en la Estrategia de Salud Familiar. Escola Anna Nery, 25(4).

Chang, H. E., \& Cho, S. H. (2016). Workplace violence and job outcomes of newly licensed nurses. Asian nursing research, 10(4), 271-276.

Correia, J. A. C. (2016). Violência no trabalho dos enfermeiros no serviço de urgência (Master's thesis).

Fernandes, L. S., Nitsche, M. J. T., \& de Godoy, I. (2017). Síndrome de burnout em profissionais de enfermagem de uma unidade de terapia intensiva Burnout syndrome in nursing professionals from an intensive care unit. Revista de Pesquisa: Cuidado é Fundamental Online, 9(2), 551-557.

Honarvar, B., Ghazanfari, N., Shahraki, H. R., Rostami, S., \& Lankarani, K. B. (2019). Violence against nurses: A Neglected and healththreatening epidemic in the university affiliated public hospitals in Shiraz, Iran. The international journal of occupational and environmental medicine, $10(3), 111$.

Lisboa, M. T. L., Moura, F. D. J. M. D., \& Reis, L. D. (2006). Violência do cotidiano e no trabalho de enfermagem: apreensões e expectativas de alunos de um curso de graduação em enfermagem do Rio de Janeiro. Escola Anna Nery, 10(1), 81-86.

Machado, C. B., Daher, D. V., Teixeira, E. R., \& Acioli, S. (2016). Violência urbana e repercussão nas práticas de cuidado no território da saúde da família [Urban violence and effect on care practices in family health strategy territories]. Revista Enfermagem UERJ, 24(5), 25458.

Marques, D. R. G., \& Silva, I. M. S. D. (2017). Violência no trabalho: Um estudo com enfermeiros/as em hospitais portugueses.

Pai, D. D., Sturbelle, I. C. S., Santos, C. D., Tavares, J. P., \& Lautert, L. (2018). Violência física e psicológica perpetrada no trabalho em saúde. Texto \& Contexto-Enfermagem, 27(1).

Pedro, D. R. C., Silva, G. K. T. D., Lopes, A. P. A. T., Oliveira, J. L. C. D., \& Tonini, N. S. (2017). Violência ocupacional na equipe de enfermagem: análise à luz do conhecimento produzido. Saúde em Debate, 41, 618-629.

Santos, C. M. D. C., Pimenta, C. A. D. M., \& Nobre, M. R. C. (2007). The PICO strategy for the research question construction and evidence search. Revista latino-americana de enfermagem, 15, 508-511.

Seo, J. W., Kang, M. R., \& Je, N. J. (2019). A study on the factors influencing burnout of psychiatric ward nurses-violence experience, violence coping, social support. Journal of The Korea Academia-Industrial Cooperation Society, 20(7), 393-405.

Silva, G. D. S. A. D., Silva, G. A. V. D., Silva, R. M. D., Andolhe, R., Padilha, K. G., \& Costa, A. L. S. (2018). Estresse e burnout em profissionais de enfermagem de unidade de terapia intensiva e semi-intensiva. Revista de Divulgação Científica Sena Aires, 7(1), 5-11.

Song, C., Wang, G., \& Wu, H. (2021). Frequency and barriers of reporting workplace violence in nurses: An online survey in China. International journal of nursing sciences, $8(1), 65-70$.

Tsukamoto, S. A. S., Galdino, M. J. Q., Robazzi, M. L. D. C. C., Ribeiro, R. P., Soares, M. H., Haddad, M. D. C. F. L., \& Martins, J. T. (2019). Violência ocupacional na equipe de enfermagem: prevalência e fatores associados. Acta Paulista de Enfermagem, 32(4), 425-432. 